\title{
Some Coefficient Problems on Bi-univalent Functions
}

\author{
Andy Liew Pik Hern ${ }^{*}$ and Aini Janteng \\ Faculty of Science and Natural Resources, Universiti Malaysia Sabah, 88400 Kota Kinabalu, Sabah, \\ Malaysia.
}

\begin{abstract}
In the open unit $\operatorname{disk} D=\{z \in \mathbb{C}:|z|<1\}$, we denote $A$ be the class of all analytic functions $f(z)$.We consider conditions $f(0)=0$ and $f^{\prime}(0)=1$ so called normalized condition and denote $\sum$ as the class of bi-univalent functions defined in $D$. If both $f(z)$ and $f^{-1}(f(z))$ are univalent in $D$, we say that a function $f \in A$ to be bi-univalent in $D$. In this paper, some subclasses of bi-univalent functions are introduced. Coefficient estimates on $\left|a_{2}\right|$ and $\left|a_{3}\right|$ are determined. In addition, the upper bounds of the Fekete-Szegö functional are also obtained.
\end{abstract}

Keywords: analytic functions, bi-univalent functions, coefficient estimates, Fekete-Szegö functional

\section{INTRODUCTION}

In this paper, the class of functions $f(z)$ which are analytic in the open unit disk $D=\{z \in \mathbb{C}:|z|<1\}$ in the form of

$$
f(z)=z+\sum_{n=2}^{\infty} a_{n} z^{n} \quad(z \in D)
$$

is denoted by $A$. We denote $S \subset A$ which are univalent in $D$ as well. According (Duren, 1983), the Koebe one-quarter theorem showed the image of $D$ under every univalent functions $f$ in $S$ contains a disk of radius 1/4. So, an inverse for every univalent function $f$ can be defined as

and

$$
f^{-1}(f(z))=z
$$

$$
f\left(f^{-1}(w)\right)=w, \quad\left(|w|<r_{o}(f), r_{o}(f) \geq 1 / 4\right) .
$$

Thus, for a function $f \in A$, if both $f$ and $f^{-1}$ are univalent in $D$, this function is bi-univalent function. The notation of bi-univalent is $\sum$.

Motivated by the previous works, for example (Shanmugam \& Sivasubramanian, 2005), (Janteng et al., 2006), (Aouf et al., 2013), (Zaprawa, 2014) and (Altinkaya \& Yalcin, 2017),we consider the following subclasses of $\sum$.

Definition 1. A function $f(z) \in \sum$ in $(1)$ is said to be in the class $A_{\Sigma}(\alpha, \lambda)$ with $0<\alpha \leq 1$ and $\lambda \geq 0$ if: $f \in \sum$ and

$$
\left|\arg \left(\frac{\lambda z^{2} f^{\prime \prime}(z)}{f(z)}+\frac{z f^{\prime}(z)}{f(z)}\right)\right|<\frac{\alpha \pi}{2}, \quad z \in D
$$

and

$$
\left|\arg \left(\frac{\lambda w^{2} g^{\prime \prime}(w)}{g(w)}+\frac{w g^{\prime}(w)}{g(w)}\right)\right|<\frac{\alpha \pi}{2}, \quad w \in D
$$

where

$$
\begin{aligned}
& g(w)=w-a_{2} w^{2}+\left(2 a_{2}^{2}-a_{3}\right) w^{3} \\
& -\left(5 a_{2}^{3}-5 a_{2} a_{3}+a_{4}\right) w^{4}+\ldots
\end{aligned} .
$$

Definition 2. A function $f(z) \in \sum$ in (1) is said to be in class $A_{\Sigma}(\beta, \lambda)$ with $0 \leq \beta<1$ and $\lambda \geq 0$ if:

$\operatorname{Re}\left(\frac{\lambda z^{2} f^{\prime \prime}(z)}{f(z)}+\frac{z f^{\prime}(z)}{f(z)}\right)>\beta$

and

$$
\operatorname{Re}\left(\frac{\lambda w^{2} g^{\prime \prime}(w)}{g(w)}+\frac{w g^{\prime}(w)}{g(w)}\right)>\beta .
$$

Definition 3. A function $f(z) \in \sum$ in (1) is said to be in the class $B_{\Sigma}(\alpha, \lambda)$ with $0<\alpha \leq 1$ and $\lambda \geq 0$ if: $f \in \sum$ and

$\left|\arg \left(\frac{\left(\lambda z^{2} f^{\prime \prime}(z)+z f^{\prime}(z)\right)^{\prime}}{f^{\prime}(z)}\right)\right|<\frac{\alpha \pi}{2}, \quad z \in D(7)$ 
and

$\left|\arg \left(\frac{\left(\lambda w^{2} g^{\prime \prime}(w)+w g^{\prime}(w)\right)^{\prime}}{g^{\prime}(w)}\right)\right|<\frac{\alpha \pi}{2}, \quad w \in D$.(8)

Definition 4. A function $f(z) \in \sum$ in (1) is said to be in class $B_{\Sigma}(\beta, \lambda)$ with $0 \leq \beta<1$ and $\lambda \geq 0$ if:

$\operatorname{Re}\left(\frac{\left(\lambda z^{2} f^{\prime \prime}(z)+z f^{\prime}(z)\right)^{\prime}}{f^{\prime}(z)}\right)>\beta$

and

$\operatorname{Re}\left(\frac{\left(\lambda w^{2} g^{\prime \prime}(w)+w g^{\prime}(w)\right)^{\prime}}{g^{\prime}(w)}\right)>\beta$.

This paper obtained the upper bound for coefficients $\left|a_{2}\right|$ and $\left|a_{3}\right|$ and Fekete-Szegö functional for $f$ in the subclasses of $\sum$.

\section{METHODS}

The following lemmas are required to get the main results.

Lemma 1. (Duren, 1983) If $p \in P$ then $\left|p_{k}\right| \leq 2$ for each $k$, where $P$ is the family of all functions $p$ analytic in $D$, $\operatorname{Re}(p(z))>0, \quad p(z)=1+p_{1} z+p_{2} z^{2}+p_{3} z^{3}+\cdots$ for $z \in D$.

Lemma 2. (Zaprawa, 2014) Let $k, l \in \mathbb{R}$ and $z_{1}, z_{2} \in \mathbb{C}$. If $\left|z_{1}\right|<R$ and $\left|z_{2}\right|<R$ then

$\left|(k+l) z_{1}+(k-l) z_{2}\right| \leq\left\{\begin{array}{ll}2|k| R & \text { for }|k| \geq|l| \\ 2|l| R & \text { for }|k| \leq|l|\end{array}\right.$.

\section{RESULTS}

The main result for $f \in A_{\Sigma}(\alpha, \lambda)$ is stated as follows.

Theorem 1. Let $f$ in (1) be in the class $A_{\Sigma}(\alpha, \lambda)$ where $0<\alpha \leq 1$ and $\lambda \geq 0$. Then

$$
\left|a_{2}\right| \leq \frac{2 \alpha}{\sqrt{4 \lambda[\lambda(1-\alpha)+\alpha+1]+\alpha+1}}
$$

and

$$
\left|a_{3}\right| \leq \frac{4 \alpha^{2}}{(2 \lambda+1)^{2}}+\frac{\alpha}{3 \lambda+1} .
$$

$$
\frac{\left(\lambda z^{2} f^{\prime \prime}(z)+z f^{\prime}(z)\right)^{\prime}}{f^{\prime}(z)}=[p(z)]^{\alpha}
$$

and

$$
\frac{\left(\lambda w^{2} g^{\prime \prime}(w)+w g^{\prime}(w)\right)^{\prime}}{g^{\prime}(w)}=[q(w)]^{a}
$$

where $p(z)$ and $q(w)$ in $P$ have the forms $p(z)=1+p_{1} z+p_{2} z^{2}+\ldots$

and

$q(w)=1+q_{1} w+q_{2} w^{2}+q_{3} w^{3}+\ldots$ respectively.

From (1), we may get

$\frac{\lambda z^{2} f^{\prime \prime}(z)+z f^{\prime}(z)}{f(z)}$

$=1+a_{2}(2 \lambda+1) z+\left[2 a_{3}(3 \lambda+1)-a_{2}^{2}(2 \lambda+1)\right] z^{2}+\ldots$

and from (4) we can get

$\frac{\lambda w^{2} g^{\prime \prime}(w)+w g^{\prime}(w)}{g(w)}$

$=1-a_{2}(2 \lambda+1) w+\left[\begin{array}{l}2 a_{3}\left(2 a_{2}^{2}-a_{3}\right)(3 \lambda+1) \\ -a_{2}^{2}(2 \lambda+1)\end{array}\right] w^{2}+\ldots$

Hence, equations (17) and (18) give

$1+a_{2}(2 \lambda+1) z+\left[2 a_{3}(3 \lambda+1)-a_{2}^{2}(2 \lambda+1)\right] z^{2}+.$.

$=1+\alpha p_{1} z+\left[\alpha p_{2}+\frac{\alpha(\alpha-1)}{2} p_{1}^{2}\right] z^{2}+\ldots$

and

$1-a_{2}(2 \lambda+1) w+\left[\begin{array}{l}2 a_{3}\left(2 a_{2}^{2}-a_{3}\right)(3 \lambda+1) \\ -a_{2}^{2}(2 \lambda+1)\end{array}\right] w^{2}+\ldots$

$=1+\alpha q_{1} w+\left[\alpha q_{2}+\frac{\alpha(\alpha-1)}{2} q_{1}^{2}\right] w^{2}+\ldots$

Next, by suitably comparing coefficients of $z$ and $z^{2}$ in (17) and comparing coefficients of $w$ and $w^{2}$ in (18), we get

$$
a_{2}(2 \lambda+1)=\alpha p_{1},
$$

$2 a_{3}(3 \lambda+1)-a_{2}^{2}(2 \lambda+1)=\alpha p_{2}+\frac{\alpha(\alpha-1)}{2} p_{1}{ }^{2}$,

$$
-a_{2}(2 \lambda+1)=\alpha q_{1},
$$

$2\left(2 a_{2}^{2}-a_{3}\right)(3 \lambda+1)-a_{2}^{2}(2 \lambda+1)$

$=\alpha q_{2}+\frac{\alpha(\alpha-1)}{2} q_{1}^{2}$

By dividing (19) and (21), we get

$$
p_{1}=-q_{1} \text {. }
$$

Next, by adding the square of equations (19) and (21), we may get

$2 a_{2}^{2}(2 \lambda+1)^{2}=\alpha^{2}\left(p_{1}^{2}+q_{1}^{2}\right)$.

Now, by adding of equations (20) and (22), we find that

Proof. It follows from (2) and (3) that 
$2 a_{3}(3 \lambda+1)-a_{2}^{2}(2 \lambda+1)+4 \alpha_{2}^{2}(3 \lambda+1)$

$-2 a_{3}(3 \lambda+1)-a_{2}^{2}(2 \lambda+1)$

$=\alpha\left(p_{2}+q_{2}\right)+\frac{\alpha(\alpha-1)}{2}\left(p_{1}^{2}+q_{1}^{2}\right)$

Then, from (23), by replacing $p_{1}^{2}+q_{1}^{2}$ into equation (24), we obtain

$a_{2}^{2}=\frac{\alpha^{2}\left(p_{2}+q_{2}\right)}{4 \lambda[\lambda(1-\alpha)+\alpha+1]+\alpha+1}$

By applying triangle inequality and Lemma 1.1 for the coefficients $p_{2}$ and $q_{2}$ into equation (25), we finally get:

$$
\left|a_{2}\right| \leq \frac{2 \alpha}{\sqrt{4 \lambda[\lambda(1-\alpha)+\alpha+1]+\alpha+1}} .
$$

This gives the bound on $\left|a_{2}\right|$ in (11). Next, to find $\left|a_{3}\right|$, by subtracting (22) from (20), we get

$$
\begin{aligned}
& 4 a_{3}(3 \lambda+1)-4 a_{2}^{2}(3 \lambda+1) \\
& =\alpha\left(p_{2}-q_{2}\right)+\frac{\alpha(\alpha-1)}{2}\left(p_{1}^{2}-q_{1}^{2}\right)
\end{aligned}
$$

Since $p_{1}=-q_{1}$ then $p_{1}^{2}=q_{1}^{2}$. Next, we have

$$
a_{3}=a_{2}^{2}+\frac{\alpha\left(p_{2}-q_{2}\right)}{4(3 \lambda+1)}
$$

From (23), we can get $a_{2}^{2}=\frac{\alpha^{2}\left(p_{1}^{2}+q_{1}^{2}\right)}{2(2 \lambda+1)^{2}}$.

Thus, by substituting $a_{2}{ }^{2}=\frac{\alpha^{2}\left(p_{1}^{2}+q_{1}^{2}\right)}{2(2 \lambda+1)^{2}}$ into equation (26), we obtain

$$
a_{3}=\frac{\alpha^{2}\left(p_{1}^{2}+q_{1}^{2}\right)}{2(2 \lambda+1)^{2}}+\frac{\alpha\left(p_{2}-q_{2}\right)}{4(3 \lambda+1)}
$$

Once again, applying triangle inequality and Lemma 1.1 for the coefficients $p_{1}, p_{2}, q_{1}$ and $q_{2}$ into equation (27), we get:

$$
\left|a_{3}\right| \leq \frac{4 \alpha^{2}}{(2 \lambda+1)^{2}}+\frac{\alpha}{3 \lambda+1} .
$$

Theorem 1 is completely proven.

Taking $\lambda=0$ in Theorem 1, we obtain the following corollary.

Corollary 1 (Murugusundaramoorthy \& Magesh, 2009)Let $f(z)$ in (1) be in the class $S S_{\Sigma}{ }^{*}(\alpha)$ and $0<\alpha \leq 1$. Then

and

$$
\left|a_{2}\right| \leq \frac{2 \alpha}{\sqrt{\alpha+1}}
$$

$$
\left|a_{3}\right| \leq 4 \alpha^{2}+\alpha
$$

Next, we obtained Theorem 2, Theorem 3 and Theorem4.
Theorem 2 Let $f$ in (1) be in the class $A_{\Sigma}(\beta, \lambda)$ where $0 \leq \beta<1$ and $\lambda \geq 0$. Then

$$
\left|a_{2}\right| \leq \sqrt{\frac{2(1-\beta)}{4 \lambda+1}}
$$

and

$$
\left|a_{3}\right| \leq \frac{4(1-\beta)^{2}}{(2 \lambda+1)^{2}}+\frac{1-\beta}{3 \lambda+1}
$$

Taking $\lambda=0$ in Theorem 2, we obtain the following corollary.

Corollary 2 (Murugusundaramoorthy \& Magesh, 2009)Let $f(z)$ in (1) be in the class $S_{\Sigma}^{*}(\beta)$ and $0 \leq \beta<1$. Then

$$
\left|a_{2}\right| \leq \sqrt{2(1-\beta)}
$$

and

$$
\left|a_{3}\right| \leq 4(1-\beta)^{2}+1-\beta \text {. }
$$

Theorem 3 Let $f$ in (1) be in the class $B_{\Sigma}(\alpha, \lambda)$ where $0<\alpha \leq 1$ and $\lambda \geq 0$. Then

$$
\left|a_{2}\right| \leq \frac{\alpha}{\sqrt{\alpha(5 \lambda+1)+(1-\alpha)(2 \lambda+1)^{2}}} \text { (3o) }
$$

and

$$
\left|a_{3}\right| \leq \frac{\alpha^{2}}{(2 \lambda+1)^{2}}+\frac{\alpha}{3(3 \lambda+1)}
$$

Taking $\lambda=0$ in Theorem 3, we obtain the following corollary.

Corollary 3 (Zaprawa, 2014) Let $f(z)$ in (1) be in the class $B_{\Sigma}(\alpha)$ and $0<\alpha \leq 1$. Then

$$
\left|a_{2}\right| \leq \alpha
$$

and

$$
\left|a_{3}\right| \leq \alpha^{2}+\frac{\alpha}{3}
$$

Theorem 4 Let $f$ in (1) be in the class $B_{\Sigma}(\beta, \lambda)$ where $0<\alpha \leq 1$ and $\lambda \geq 0$. Then

$$
\left|a_{2}\right| \leq \sqrt{\frac{1-\beta}{5 \lambda+1}}
$$

and

$$
\left|a_{3}\right| \leq \frac{2(1-\beta)^{2}}{(2 \lambda+1)^{2}}+\frac{1-\beta}{3(3 \lambda+1)} .
$$

Taking $\lambda=0$ in Theorem 4 , we obtain the following corollary.

Corollary 4 Let $f(z)$ in (1) be in the class $B_{\Sigma}(\beta)$ and $0 \leq \beta<1$. Then

$$
\left|a_{2}\right| \leq \sqrt{1-\beta}
$$


and

$$
\left|a_{3}\right| \leq 2(1-\beta)^{2}+\frac{1}{3}(1-\beta) \text {. }
$$

Our second main results for the classes $A_{\Sigma}(\alpha, \lambda)$, $A_{\Sigma}(\beta, \lambda), B_{\Sigma}(\alpha, \lambda)$ and $B_{\Sigma}(\beta, \lambda)$ are given by Theorem 5 , Theorem 6, Theorem 7 and Theorem 8.

Theorem 5 If $f \in A_{\Sigma}(\alpha, \lambda), 0<\alpha \leq 1, \lambda \geq 0$ and $\mu \in \mathbb{R}$, then

$\left|a_{3}-\mu a_{2}^{2}\right|$

$$
\leq\left\{\begin{array}{rr}
\frac{4 \alpha^{2}}{2 \alpha(4 \lambda+1)-(\alpha-1)(2 \lambda+1)^{2}}|1-\mu| & \text { for } 4 \alpha(3 \lambda+1)|(1-\mu)| \\
& \geq 2 \alpha(4 \lambda+1)-(\alpha-1) \\
\frac{\alpha}{3 \lambda+1} & \text { for } 4 \alpha(3 \lambda+1)|(1-\mu)| \\
& \leq 2 \alpha(4 \lambda+1)-(\alpha-1)(2 \lambda+1)^{2}
\end{array}\right.
$$

Proof. Let $f$ given by (1) be in $A_{\Sigma}(\alpha, \lambda), \quad 0<\alpha \leq 1$, $\lambda \geq 0$ and $\mu \in \mathbb{R}$.

By adding of equations (20) and (22), we find that

$2 a_{2}^{2}(4 \lambda+1)$

$=\alpha\left(p_{2}+q_{2}\right)+\frac{1}{2} \alpha(\alpha-1)\left(p_{1}^{2}+q_{1}^{2}\right)^{(35)}$

Next, by subtracting of equations (22) from (20), we get $a_{3}=a_{2}^{2}+\frac{\alpha\left(p_{2}-q_{2}\right)}{4(3 \lambda+1)}$

From equation (23), we have

$$
p_{1}^{2}+q_{1}^{2}=\frac{2 a_{2}^{2}(2 \lambda+1)^{2}}{\alpha^{2}}
$$

By substituting equation (37) into equation (35), yields

$$
a_{2}^{2}=\frac{\alpha^{2}}{2 \alpha(4 \lambda+1)-(\alpha-1)(2 \lambda+1)^{2}}\left(p_{2}+q_{2}\right)
$$

By substituting equation (38) into equation (36),

$$
\begin{aligned}
& =\left[\frac{\alpha^{2}}{2 \alpha(4 \lambda+1)-(\alpha-1)(2 \lambda+1)^{2}}\right]\left(p_{2}+q_{2}\right) \\
& \quad+\frac{\alpha}{4(3 \lambda+1)}\left(p_{2}-q_{2}\right)
\end{aligned}
$$

Thus, from equations (38) and (39), we obtain $a_{3}-\mu a_{2}^{2}$

$$
\begin{aligned}
& =\left[\frac{\alpha^{2}}{2 \alpha(4 \lambda+1)-(\alpha-1)(2 \lambda+1)^{2}}\right]\left(p_{2}+q_{2}\right)+\frac{\alpha}{4(3 \lambda+1)}\left(p_{2}-q_{2}\right) \\
& -\mu\left[\frac{\alpha^{2}}{2 \alpha(4 \lambda+1)-(\alpha-1)(2 \lambda+1)^{2}}\left(p_{2}+q_{2}\right)\right] \\
& =h(\alpha, \lambda)(1-\mu)\left(p_{2}+q_{2}\right)+\frac{\alpha}{4(3 \lambda+1)}\left(p_{2}-q_{2}\right)
\end{aligned}
$$

where $\quad h(\alpha, \lambda)=\frac{\alpha^{2}}{2 \alpha(4 \lambda+1)-(\alpha-1)(2 \lambda+1)^{2}} \quad$ is nonnegative.

Hence,

$$
\begin{aligned}
& a_{3}-\mu a_{2}{ }^{2} \\
& =h(\alpha, \lambda)(1-\mu) p_{2}+h(\alpha, \lambda)(1-\mu) q_{2} \\
& +\frac{\alpha}{4(3 \lambda+1)} p_{2}-\frac{\alpha}{4(3 \lambda+1)} q_{2} \\
& =\left[h(\alpha, \lambda)(1-\mu)+\frac{\alpha}{4(3 \lambda+1)}\right] p_{2} \\
(2 \lambda+1)^{2}, & +\left[h(\alpha, \lambda)(1-\mu)-\frac{\alpha}{4(3 \lambda+1)}\right] q_{2}
\end{aligned}
$$

From Lemma 1 and Lemma 2, we obtain

$\left|a_{3}-\mu a_{2}^{2}\right|$

$$
\begin{aligned}
& =\left|\begin{array}{l}
\left.h(\alpha, \lambda)(1-\mu)+\frac{\alpha}{4(3 \lambda+1)}\right] p_{2} \\
+\left[h(\alpha, \lambda)(1-\mu)-\frac{\alpha}{4(3 \lambda+1)}\right] q_{2}
\end{array}\right| \\
& \leq\left\{\begin{array}{c}
2|h(\alpha, \lambda)(1-\mu)|(2) \text { for }|h(\alpha, \lambda)(1-\mu)| \\
\geq \frac{\alpha}{4(3 \lambda+1)} \\
2\left|\frac{\alpha}{4(3 \lambda+1)}\right|(2) \text { for }|h(\alpha, \lambda)(1-\mu)| \leq \frac{\alpha}{4(3 \lambda+1)}
\end{array}\right.
\end{aligned}
$$

$$
=\left\{\begin{array}{c}
4 h(\alpha, \lambda)|1-\mu| \text { for } h(\alpha, \lambda)|(1-\mu)| \geq \frac{\alpha}{4(3 \lambda+1)} \\
\frac{\alpha}{3 \lambda+1} \text { for } h(\alpha, \lambda)|(1-\mu)| \leq \frac{\alpha}{4(3 \lambda+1)}
\end{array}\right.
$$

Theorem 5 is completely proven.

Taking $\mu=0$ and $\lambda=0$ in Theorem 5 , we obtain the following corollary.

Corollary 5 If $f(z)$ in equation (1) be in the class $S S_{\Sigma}^{*}(\alpha), 0<\alpha \leq 1$, then

$$
\left|a_{3}\right| \leq\left\{\begin{array}{ll}
\alpha & \text { for } 0<\alpha \leq \frac{1}{3} \\
\frac{4 \alpha^{2}}{\alpha+1} & \text { for } \frac{1}{3} \leq \alpha \leq 1
\end{array} .\right.
$$

The result in Corollary 5 is similar to Corollary 10 in (Zaprawa, 2014) if $\lambda=0$.

Putting $\alpha=1$ in Corollary 5, we will get the following corollary.

Corollary 6 (Zaprawa, 2014) If $f(z)$ in equation (1) 
be in the class $S S_{\Sigma}{ }^{*}(1)$, then

$$
\left|a_{3}\right| \leq 2 \text {. }
$$

Next, we obtained Theorem 6, Theorem 7 and Theorem 8 as follows.

Theorem 6 If $f \in A_{\Sigma}(\beta, \lambda), \quad 0 \leq \beta<1, \quad \lambda \geq 0$ and $\mu \in \mathbb{R}$, then

$\left|a_{3}-\mu a_{2}^{2}\right| \leq\left\{\begin{array}{cc}\frac{2(1-\beta)}{(4 \lambda+1)}|1-\mu| & \text { for } 2(3 \lambda+1)|1-\mu| \geq 4 \lambda+1, \\ \frac{1-\beta}{3 \lambda+1} & \text { for } 2(3 \lambda+1)|1-\mu| \leq 4 \lambda+1 .\end{array}\right.$

Taking $\mu=0$ and $\lambda=0$ in Theorem 6, we obtain the following corollary.

Corollary 7 If $f(z)$ in equation (1) be in the class $S_{\Sigma}^{*}(\beta), 0 \leq \beta<1$, then

$$
\left|a_{3}\right| \leq 2(1-\beta) \text {. }
$$

The result in Corollary 7 is similar to Corollary 11 in (Zaprawa, 2014) if $\lambda=0$. $\left|a_{3}-\mu a_{2}^{2}\right| \leq \begin{cases}\frac{1-\beta}{5 \lambda+1}|1-\mu| & \text { for } 3(3 \lambda+1)|(1-\mu)| \geq 5 \lambda+1, \\ \frac{1-\beta}{3(3 \lambda+1)} & \text { for } 3(3 \lambda+1)|(1-\mu)| \leq 5 \lambda+1 .\end{cases}$

(42)

Taking $\mu=0$ and $\lambda=0$ in Theorem 8 , we obtain the following corollary.

Corollary 10 (Zaprawa, 2014) If $f(z)$ in equation (1) be in the class $B_{\Sigma}(\beta), 0 \leq \beta<1$, then

$$
\left|a_{3}\right| \leq 1-\beta \text {. }
$$

\section{SUMMARY}

In conclusion, coefficient estimates on $\left|a_{2}\right|$ and $\left|a_{3}\right|$ are obtained for some subclasses of bi-univalent functions. Furthermore, the upper bounds of the Fekete-Szegö functional are also obtained.

Theorem 7 If $f \in B_{\Sigma}(\alpha, \lambda), \quad 0<\alpha \leq 1$,

$\lambda \geq 0$ and $\mu \in \mathbb{R}$, then

$\left|a_{3}-\mu a_{2}{ }^{2}\right| \leq\left\{\begin{aligned} \frac{\alpha^{2}}{\alpha(5 \lambda+1)+(1-\alpha)(2 \lambda+1)^{2}}|1-\mu| & \text { for } 3 \alpha(3 \lambda+1)|(1-\mu)| \\ & \geq \alpha(5 \lambda+1)+(1-\alpha)(2 \lambda+1)^{2}, \\ \frac{\alpha}{3(3 \lambda+1)} & \text { for } 3 \alpha(3 \lambda+1)|(1-\mu)| \leq \alpha(5 \lambda+1)+(1-\alpha)(2 \lambda+1)^{2} .\end{aligned}\right.$

Taking $\mu=0$ and $\lambda=0$ in Theorem 7, we obtain the following corollary.

Corollary 8 If $f(z)$ in equation (1) be in the class $B_{\Sigma}(\alpha), 0<\alpha \leq 1$, then

$$
\left|a_{3}\right| \leq\left\{\begin{array}{ll}
\frac{1}{3} \alpha & \text { for } 0<\alpha \leq \frac{1}{3} \\
\alpha^{2} & \text { for } \frac{1}{3} \leq \alpha \leq 1
\end{array}\right. \text {. }
$$

The result in Corollary 8 is similar to Corollary 17 in (Zaprawa, 2014) if $\lambda=0$.

Putting $\alpha=1$ in Corollary 8 , we will get the following corollary.

Corollary 9 If $f(z)$ in equation (1) be in the class $B_{\Sigma}(1)$, then

$$
\left|a_{3}\right| \leq 1
$$

Theorem $8 \quad$ If $f \in B_{\Sigma}(\beta, \lambda), \quad 0 \leq \beta<1, \quad \lambda \geq 0$ and $\mu \in \mathbb{R}$, then 


\section{REFERENCES}

Altinkaya, Ş. \& Yalcin, S. (2017). The Feketa-Szegö problem for a general class of bi-univalent functions satisfying subordinate conditions. Sahand Communications in Mathematical Analysis, 5(1): 1-7.

Aouf, M. K., El-Ashwah, R. M. \& Zayed, H. M. (2013). Fekete-Szegö inequalities for certain class of meromorphic functions. Journal of the Egyptian Mathematical Society, 21:197-200.

Duren, P. L. (1983). Univalent functions. Grundlehren der mathematicschen Wissenschanften, 259. New York: Springer-Verlag.
Janteng, A., Halim, S. A. \& Darus, M. (2006). Fekete-Szegö problem for certain subclass of quasi-convex functions. Int. J. Contemp. Math. Sci., 1(1):45-51.

Murugusundaramoorthy, G. \& Magesh, N. (2009). Coefficient inequalities for certain classes of analytic functions associated with Hankel determinant. Bulletin of Mathematical Analysis and Applications, 1(3): 85-89.

Shanmugam, T. \& Sivasubramanian, S. (2005). On the Fekete-Szegö problem for some subclasses of analytic functions. J. Inequal. Pure Appl. Math., 6(3)(2005): 115 .

Zaprawa, P. (2014). Estimates of Initial Coefficients for BiUnivalent Function. Abstract and Applied Analysis, 2014 . 